\title{
Sharp inequalities for the coefficients of concave schlicht functions
}

\author{
F. G. Avkhadiev, Ch. Pommerenke and K.-J. Wirths
}

Abstract. Let $D$ denote the open unit disc and let $f: D \rightarrow \mathbb{C}$ be holomorphic and injective in $D$. We further assume that $f(D)$ is unbounded and $\mathbb{C} \backslash f(D)$ is a convex domain. In this article, we consider the Taylor coefficients $a_{n}(f)$ of the normalized expansion

$$
f(z)=z+\sum_{n=2}^{\infty} a_{n}(f) z^{n}, \quad z \in D,
$$

and we impose on such functions $f$ the second normalization $f(1)=\infty$. We call these functions concave schlicht functions, as the image of $D$ is a concave domain. We prove that the sharp inequalities

$$
\left|a_{n}(f)-\frac{n+1}{2}\right| \leq \frac{n-1}{2}, \quad n \geq 2,
$$

are valid. This settles a conjecture formulated in [2].

Mathematics Subject Classification (2000). 30C50, 30C45.

Keywords. Taylor coefficients, concave schlicht functions, slit mappings.

Let $D$ denote the open unit disc and let $f: D \rightarrow \mathbb{C}$ be holomorphic and injective in $D$. In the last century, much research in geometric function theory was motivated by the Bieberbach conjecture, which assures that for such functions $f$ the sharp inequalities $\left|a_{n}(f)\right| \leq n, n \geq 2$, are valid, if $f$ is normalized by the expansion

$$
f(z)=z+\sum_{n=2}^{\infty} a_{n}(f) z^{n}, \quad z \in D .
$$

Long before the proof of the Bieberbach conjecture by de Branges in 1985, Löwner had obtained in 1917 that the inequalities $\left|a_{n}(f)\right| \leq 1$ hold for convex functions $f$.

In the present paper, we prove sharp inequalities bridging the gap between these two theorems. We consider functions $f$ of the above type for which $f(D)$ is unbounded and $\mathbb{C} \backslash f(D)$ is a convex set. We impose on these functions $f$ the second normalization $f(1)=\infty$ and we call such functions concave schlicht functions. In [1] it was conjectured that for concave schlicht functions the inequalities $\left|a_{n}(f)\right| \geq 1$, 
$n \geq 2$, are valid. This conjecture was proved in [2] and we also conjectured in the same article that the inequalities

$$
\left|a_{n}(f)-\frac{n+1}{2}\right| \leq \frac{n-1}{2}
$$

are valid for concave schlicht functions $f$ and for $n \geq 2$. For $\theta \in[0,2 \pi] \backslash\{\pi\}$ the conformal maps

$$
f_{\theta}(z)=\frac{1}{2\left(1+e^{i \theta}\right)}\left(\left(\frac{1+e^{i \theta} z}{1-z}\right)^{2}-1\right)=\frac{z-\frac{1}{2}\left(1-e^{i \theta}\right) z^{2}}{(1-z)^{2}}
$$

map $D$ onto the complex plane minus a (possibly skew) halfline, whereas

$$
f_{\pi}(z)=\frac{z}{1-z}
$$

maps $D$ onto a half plane. It is easy to check that equality holds in (1) for every $n$ if $f=f_{\theta}, \theta \in[0,2 \pi]$.

Concerning the history of the conjecture (1) we mention that (1) is the limiting case of another conjecture from [2] that seems to be more difficult to prove than (1). That conjecture concerns functions $f: D \rightarrow \overline{\mathbb{C}}$ meromorphic and injective in $D$ such that $\overline{\mathbb{C}} \backslash f(D)$ is convex, $f$ has a simple pole at the point $p \in(0,1)$ and a normalized expansion

$$
f(z)=z+\sum_{n=2}^{\infty} a_{n}(f) z^{n}, \quad|z|<p .
$$

These functions are called concave univalent functions with pole $p$. The family of all such functions is denoted by $\operatorname{Co}(p)$. It was conjectured in [2] that

$$
\left|a_{n}(f)-\frac{1-p^{2 n+2}}{p^{n-1}\left(1-p^{4}\right)}\right| \leq \frac{p^{2}\left(1-p^{2 n-2}\right)}{p^{n-1}\left(1-p^{4}\right)}
$$

for $f \in \operatorname{Co}(p), p \in(0,1)$ and $n \geq 2$. The limiting case for $p \rightarrow 1$ of (4) is (1) and any concave schlicht function can be approximated by concave univalent functions with pole in the unit disc (compare [1]).

J. Miller proved (4) for $n=2$ in [7]. A. E. Livingston showed in [5] that

$$
\operatorname{Re}\left(a_{3}(f)\right) \geq \frac{1+p^{6}}{p^{2}\left(1+p^{2}\right)}
$$

for $f \in \operatorname{Co}(p)$ and conjectured for $f \in \operatorname{Co}(p)$ and $n \geq 2$ the validity of the inequality

$$
\operatorname{Re}\left(a_{n}(f)\right) \geq \frac{1+p^{2 n}}{p^{n-1}\left(1+p^{2}\right)} .
$$


The case $n=3$ of (4) was proved in [2] and the cases $n=4$ and $n=5$ in [9]. According to the above observation this implies that (1) has been proved for $n=2,3,4,5$.

In the sequel we will show that (1) holds for all $n \geq 2$. This is the content of the following theorem.

Theorem. For any concave schlicht function $f(z)=z+\cdots$ and any $n \geq 2$ the inequality (1) is valid. Equality in (1) is attained if and only if $f=f_{\theta}$ for some $\theta \in[0,2 \pi]$.

The proof will show that (1) also holds for all functions in the closed convex hull of the family of concave schlicht functions. First, we reduce our problem to a question concerning the convex hull of certain products. This question has been studied by Brickman, Hallenbeck, MacGregor, and Wilken in [3]. Their results allow us to prove the following proposition.

Proposition. If $f(z)=z+\cdots$ is a concave schlicht function then

$$
f^{\prime}(z)=\frac{1+z \omega(z)}{(1-z)^{3}}, \quad z \in D,
$$

where $\omega: D \rightarrow \bar{D}$ is holomorphic.

Proof. First we observe that the boundary of $f(D)$ may be approximated by concave polygons $C_{m}, m \in \mathbb{N}$, that form an angle $\pi \alpha, \alpha \in[1,2]$, at infinity and have $m$ vertices $z_{k} \in \mathbb{C}, k=1, \ldots, m$, such that $\pi \beta_{k}, k=1, \ldots, m, 0<\beta_{k} \leq 1$, is the change in the direction of $C_{m}$ at the vertex $z_{k}$. In the case $\alpha=1$, we only have to consider a straight line.

Now we prove the proposition for the concave schlicht functions that map $D$ onto the concave domain bounded by $C_{m}$. According to the Schwarz-Christoffel formula for these maps $f$ we get the existence of $m$ preimages

$$
\exp \left(-i t_{k}\right), \quad k=1, \ldots, m, 0<t_{1}<\cdots<t_{m}<2 \pi,
$$

for the vertices of $C_{m}$ such that

$$
f^{\prime}(z)=(1-z)^{-\alpha-1} \prod_{k=1}^{m}\left(1-e^{i t_{k}} z\right)^{\beta_{k}},
$$

where

$$
\sum_{k=1}^{m} \beta_{k}=\alpha-1 .
$$


Now we consider the function

$$
g(z)=\frac{1}{f^{\prime}(z)(1-z)^{3}}=\frac{1}{(1-z)^{2-\alpha} \prod_{k=1}^{m}\left(1-e^{i t_{k}} z\right)^{\beta_{k}}} .
$$

In Lemma 1 and Theorem 1 of [3] it was shown that products of the form

$$
g(z)=\prod_{k=1}^{m+1}\left(1-e^{i t_{k}} z\right)^{-\delta_{k}}, \quad \sum_{k=1}^{m+1} \delta_{k}=1, \quad \delta_{k}>0,
$$

have a representation

$$
g(z)=\int_{\partial D} \frac{d \mu(x)}{1-x z}
$$

where $\mu$ is a probability measure on $\partial D$. Hence,

$$
\operatorname{Re}(g(z))>\frac{1}{2}, \quad z \in D .
$$

Therefore, there exists a holomorphic function $\omega: D \rightarrow \bar{D}$ such that

$$
g(z)=\frac{1}{1+z \omega(z)}
$$

which yields the representation formula (5) for the polygon mappings.

To complete the proof we use that the set of functions

$$
\left\{\frac{1+z \omega(z)}{(1-z)^{3}} \mid \omega: D \rightarrow \bar{D} \text { holomorphic }\right\} .
$$

is convex and closed in the topology of uniform convergence on compact subsets of $D$. Therefore it contains not only the derivatives of the concave polygon mappings, but also the derivatives of all concave schlicht functions. This proves the proposition.

Proof of the theorem. We write $f^{\prime}$ in the form (5). Let

$$
\omega(z)=\sum_{k=0}^{\infty} c_{k} z^{k}
$$

Inserting (6) into (5) leads to the equations

$$
n a_{n}(f)=\frac{n(n+1)}{2}+\sum_{k=0}^{n-2} c_{k} \frac{(n-k)(n-k-1)}{2}, \quad n \geq 2 .
$$


Now we prove our theorem by showing that, for holomorphic functions $\omega: D \rightarrow \bar{D}$ with the Taylor expansion (6), the inequalities

$$
\left|\sum_{k=0}^{n-2} c_{k} \frac{(n-k)(n-k-1)}{2}\right| \leq \frac{n(n-1)}{2}
$$

are valid and that equality is attained in (7) if and only if $\omega(z) \equiv \exp (i \theta), \theta \in[0,2 \pi]$. This implies that (1) is valid even for the functions in the closed convex hull of the family of concave schlicht functions. The second part of this assertion yields that the only functions in this hull for which equality is attained in (1) are the functions $f_{\theta}$ defined in (2) and (3). Since the derivative of any concave schlicht function satisfies (5), we conclude that equality in (1) holds precisely for these functions.

To prove (7) it is sufficient to consider those functions $\omega$ that are holomorphic in the closed unit disc. For these we get

$$
\sum_{k=0}^{n-2} c_{k} \frac{(n-k)(n-k-1)}{2}=\frac{1}{2 \pi i} \int_{\partial D} \omega(z) \kappa_{n}(z) d z,
$$

where

$$
\kappa_{n}(z)=\sum_{k=0}^{n-2} \frac{(n-k)(n-k-1)}{2} z^{-k-1} .
$$

Now we replace the kernel $\kappa_{n}$ by another one that produces the same linear functional on $H^{\infty}$. To this end let

$$
P_{n}(z)=\sum_{j=0}^{n-3} \frac{(j+2)(j+1)}{2}\left(z^{j}+z^{2 n-4-j}\right)+\frac{n(n-1)}{2} z^{n-2}, \quad n \geq 2,
$$

and

$$
K_{n}(z)=z^{-n+1} P_{n}(z) .
$$

Since $\kappa_{n}$ and $K_{n}$ have the same singular parts at the origin, it is evident that

$$
\frac{1}{2 \pi i} \int_{\partial D} \omega(z) \kappa_{n}(z) d z=\frac{1}{2 \pi i} \int_{\partial D} \omega(z) K_{n}(z) d z .
$$

To consider the polynomials $P_{n}$ in more detail, we define the functions $Q_{n}, n \geq 2$, by

$$
Q_{n}(\varphi)=\sum_{j=0}^{n-3}(j+2)(j+1) \cos ((n-j-2) \varphi)+\frac{n(n-1)}{2}, \quad \varphi \in[0,2 \pi],
$$


and observe that

$$
P_{n}\left(e^{i \varphi}\right)=e^{i(n-2) \varphi} Q_{n}(\varphi), \quad \varphi \in[0,2 \pi] .
$$

Now we show that for $n \geq 2$ the inequalities

$$
Q_{n}(\varphi) \geq 1, \quad \varphi \in[0,2 \pi],
$$

are valid. For the proof of this inequality we use the fact that the generating function of the sequence $Q_{n}(\varphi), n \geq 2$, is

$$
\sum_{n=1}^{\infty} Q_{n+1}(\varphi) z^{n}=\frac{1}{2} \frac{z}{(1-z)^{3}}\left(\frac{1+e^{i \varphi} z}{1-e^{i \varphi} z}+\frac{1+e^{-i \varphi} z}{1-e^{-i \varphi} z}\right) .
$$

Representing this function by the sum of its singular parts at the poles $z_{1}=1$, $z_{2}=e^{i \varphi}$, and $z_{3}=e^{-i \varphi}$ and using the Taylor expansions of these singular parts, we obtain for $n \geq 2$ and $\varphi \in[0,2 \pi]$

$$
Q_{n}(\varphi)=\frac{2 n-1-\frac{\sin \left((2 n-1) \frac{\varphi}{2}\right)}{\sin \left(\frac{\varphi}{2}\right)}}{2(1-\cos (\varphi))}=1+\sum_{j=2}^{n-1} \frac{1-\cos (j \varphi)}{1-\cos (\varphi)} \geq 1>0 .
$$

After these preparations we can prove the inequality (7) by the following chain of relations:

$$
\begin{aligned}
\left|\frac{1}{2 \pi i} \int_{\partial D} \omega(z) K_{n}(z) d z\right| & \leq \frac{1}{2 \pi} \int_{0}^{2 \pi}\left|e^{i \varphi} K_{n}\left(e^{i \varphi}\right)\right| d \varphi\|\omega\|_{\infty} \\
& =\frac{1}{2 \pi} \int_{0}^{2 \pi} Q_{n}(\varphi) d \varphi\|\omega\|_{\infty} \leq \frac{1}{2 \pi} \int_{0}^{2 \pi} Q_{n}(\varphi) d \varphi \\
& =\frac{1}{2 \pi i} \int_{\partial D} K_{n}(z) d z=\frac{n(n-1)}{2} .
\end{aligned}
$$

We see that equality is attained everywhere if $\omega(z) \equiv 1$.

To prove the above uniqueness statement we apply the theory of extremum problems for linear functionals on $H^{p}, 1 \leq p \leq \infty$, due to Macintyre, Rogosinski, and Shapiro (see [6], [8], and Duren's book [4] on $H^{p}$ spaces, Ch. 8) to the linear functional $\Phi_{n}$ defined on $H^{\infty}$ by

$$
\Phi_{n}(\omega)=\sum_{k=0}^{n-2} c_{k} \frac{(n-k)(n-k-1)}{2} .
$$

This theory (compare in particular [4], Theorem 8.1) assures that there is a unique extremal function $\omega_{E}$ such that

$$
\max \left\{\left|\Phi_{n}(\omega)\right| \mid \omega \in H^{\infty},\|\omega\|_{\infty} \leq 1\right\}=\Phi_{n}\left(\omega_{E}\right) .
$$


The above considerations show that in our case $\omega_{E}(z) \equiv 1$. Hence, equality in (7) is attained only in the specified cases. This concludes the proof of our theorem.

We want to add that the theory of Macintyre, Rogosinski, and Shapiro and its representation in Duren's book encouraged and enabled us to find the above simple solution of the extremum problem (7).

Acknowledgement. The authors thank the Deutsche Forschungsgemeinschaft for several grants for F. G. Avkhadiev. During his research stays in Braunschweig we discussed the problems of this article.

\section{References}

[1] F. G. Avkhadiev and K.-J. Wirths, Convex holes produce lower bounds for coefficients. Complex Var. Theory Appl. 47 (2002), 553-563. Zbl 1028.30010 MR 1918558

[2] F. G. Avkhadiev, Ch. Pommerenke, and K.-J. Wirths, On the coefficients of concave univalent functions. Math. Nachr. 271 (2004), 3-9. Zbl 02093892 MR 2068879

[3] L. Brickman, D. J. Hallenbeck, T. H. MacGregor, and D. R. Wilken, Convex hulls and extreme points of families of starlike and convex mappings. Trans. Amer. Math. Soc. 185 (1973), 413-228. Zbl 0278.30021 MR 0338337

[4] P. L. Duren, Theory of $H^{p}$ spaces. Pure Appl. Math. 38, Academic Press, New York 1970. Zbl 0215.20203 MR 0268655

[5] A. E. Livingston, Convex meromorphic mappings. Ann. Polon. Math. 59 (1994), 275-291. Zbl 0811.30010 MR 1282779

[6] A. J. Macintyre and W. W. Rogosinski, Extremum problems in the theory of analytic functions. Acta Math. 82 (1950), 275-325. Zbl 0036.04503 MR 0036314

[7] J. Miller, Convex and starlike meromorphic functions. Proc. Amer. Math. Soc. 80 (1980), 607-613. Zbl 0451.30008 MR 0587937

[8] W. W. Rogosinski and H. S. Shapiro, On certain extremum problems for analytic functions. Acta Math. 90 (1953), 287-318. Zbl 0051.05604 MR 0059354

[9] K.-J. Wirths, A proof of the Livingston conjecture for the fourth and the fifth coefficient of concave univalent functions. Ann. Polon. Math. 83 (2004), 87-93. Zbl 1058.30018 MR 2041745

Received October 2, 2004

F. G. Avkhadiev, Chebotarev Research Institute, Kazan State University, 420008 Kazan, Russia

E-mail: Farit.Avhadiev@ksu.ru

Ch. Pommerenke, Institut für Mathematik, TU Berlin, 10623 Berlin, Germany

E-mail: pommeren@math.tu-berlin.de

K.-J. Wirths, Institut für Analysis und Algebra, TU Braunschweig, 38106 Braunschweig, Germany

E-mail: kjwirths@tu-bs.de 